\title{
Locally recurrent chondrosarcoma of the pelvis and limbs can only be controlled by wide local excision
}

\section{Laitinen, M. K.}

2019-03

Laitinen , M K , Parry , M C , Le Nail , L-R , Wigley , C H , Stevenson , J D \& Jeys , L M 2019

, ' Locally recurrent chondrosarcoma of the pelvis and limbs can only be controlled by wide local excision ' , The bone \& joint journal , vol. 101B , no. 3 , pp. 266-271 . https://doi.org/10.1302/0301-620X.101B3

http://hdl.handle.net/10138/313326

https://doi.org/10.1302/0301-620X.101B3.BJJ-2018-0881.R1

unspecified

publishedVersion

Downloaded from Helda, University of Helsinki institutional repository.

This is an electronic reprint of the original article.

This reprint may differ from the original in pagination and typographic detail.

Please cite the original version. 


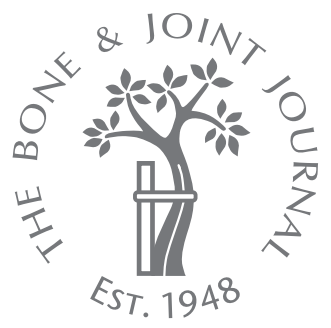

M. K. Laitinen,

M. C. Parry,

L-R. Le Nail,

C. H. Wigley,

J. D. Stevenson,

L. M. Jeys

From Royal

Orthopaedic Hospital,

Birmingham, United

Kingdom

M. K. Laitinen, MD,

Orthopaedic Surgeon

Department of Orthopaedics

and Traumatology, Helsinki

University Hospital, Helsinki,

Finland.

M. C. Parry, BSc, MBChS, MD, FRCS, Consultant in Orthopaedic Oncology and Reconstructive Surgery

I. J. Stevenson, MBChB,

BMedSci, FRCS, Consultant

in Orthopaedic Oncology and

Reconstructive Surgery

Unit of Oncology, Roya

Orthopaedic Hospital,

Birmingham, UK; Honorary

Senior Lecturer, Aston

University Medical School,

Birmingham, UK.

- L-R. Le Nail, MD,

Orthopaedic Surgeon, Unit of

Oncology, Royal Orthopaedic

Hospital, Birmingham, UK;

Centre Hospitalier Régional

Universitaire (CHU) de Tours,

Tours, France.

ㄷ. H. Wigley, $\mathrm{MBChB}$ BMedSc(Hons), Foundation

Year Doctor

L. M. Jeys, MSc, MBChB

FRCS, Professor and Consultant

Orthopaedic Surgeon

Unit of Oncology, Royal

Orthopaedic Hospital,

Birmingham, UK.

Correspondence should be sent to M. K. Laitinen; email: minna.laitinen@hus.fi

()2019 The British Editorial

Society of Bone \& Joint Surgery

doi:10.1302/0301-620X.101B3.

BJJ-2018-0881.R1 \$2.00

Bone Joint $J$

2019;101-B:266-271.

\section{Locally recurrent chondrosarcoma of the pelvis and limbs can only be controlled by wide local excision}

Aims

The purpose of this study was to investigate the potential for achieving local and systemic control after local recurrence of a chondrosarcoma of bone.

\section{Patients and Methods}

A total of 126 patients with local recurrence (LR) of chondrosarcoma (CS) of the pelvis or a limb bone were identified from a prospectively maintained database, between 1990 and $\mathbf{2 0 1 5}$ at the Royal Orthopaedic Hospital, Birmingham, United Kingdom. There were 44 female patients (35\%) and 82 male patients (65\%) with a mean age at the time of LR of 56 years (13 to 96 ). The 126 patients represented $24.3 \%$ of the total number of patients with a primary CS (519) who had been treated during this period. Clinical data collected at the time of primary tumour and LR included the site (appendicular, extremity, or pelvis); primary and LR tumour size (in centimetres); type of operation at the time of primary or LR (limb-salvage or amputation); surgical margin achieved at resection of the primary tumour and the LR; grade of the primary tumour and the LR; gender; age; and oncological outcomes, including local recurrence-free survival and disease-specific survival. A minimum two years' follow-up and complete histopathology records were available for all patients included in the study.

\section{Results}

For patients without metastases prior to or at the time of local recurrence, the diseasespecific survival after local recurrence was $62.5 \%$ and $45.5 \%$ at one and five years, respectively. After univariable analysis, significant factors predicting disease-specific survival were grade ( $p<0.001)$ and surgical margin $(p=0.044)$. After multivariable analysis, grade, increasing age at the time of diagnosis of local recurrence, and a greater time interval from primary surgery to local recurrence were significant factors for diseasespecific survival. A secondary local recurrence was seen in $26 \%$ of patients. Wide margins were a good predictor of local recurrence-free survival for subsequent recurrences after univariable analysis when compared with intralesional margins $(p=0.002)$ but marginal margins did not reach statistical significance when compared with intralesional margins $(p=0.084)$.

\section{Conclusion}

In cases of local recurrence of a chondrosarcoma of bone, we have shown that if the tumour is non-metastatic at re-staging, an increase in disease-specific survival and in local recurrence-free survival is achievable, but only by resection of the local recurrence with a wide margin.

Cite this article: Bone Joint J 2019;101-B:266-271.

Chondrosarcoma (CS) is a malignant neoplasm with cartilage differentiation. It is the second most common primary bone malignancy ${ }^{1,2}$ and is classified on its histological grade for cellularity, atypia, and pleomorphism. However, the histological grading is prone to variations in interpretation. $^{3}$ This is of importance as CS is resistant to radiotherapy and conventional chemotherapy; resection, with appropriate margins for the grade of tumour, should be the primary goal., ${ }^{2,4-6}$ What constitutes a sufficient margin is debatable. In grade $1 \mathrm{CS}$, intralesional resection with addition of adjuvant treatment, such as cementation, is a widely accepted treatment. ${ }^{7-10}$ In true grade $1 \mathrm{CS}$, 
Table I. Patient characteristics

\begin{tabular}{|c|c|}
\hline Patient characteristics & n (\%) \\
\hline Eligible patients & $126(100)$ \\
\hline \multicolumn{2}{|l|}{ Gender } \\
\hline Male & $82(65)$ \\
\hline Female & $44(35)$ \\
\hline \multicolumn{2}{|l|}{ Location } \\
\hline Pelvis & $54(43)$ \\
\hline Extremity & $69(55)$ \\
\hline Appendicular & $2(2)$ \\
\hline \multicolumn{2}{|l|}{ Grade of the local recurrence } \\
\hline Grade 1 & $11(9)$ \\
\hline Grade 2 & $35(28)$ \\
\hline Grade 3 & $16(13)$ \\
\hline Dedifferentiated & $19(15)$ \\
\hline No histology & $17(14)$ \\
\hline Not applicable & $28(22)$ \\
\hline Increase in grade from primary to local recurrence & $18(23)$ \\
\hline \multicolumn{2}{|l|}{ Surgical treatment for local recurrence } \\
\hline No surgery & $40(32)$ \\
\hline Limb salvage & $63(50)$ \\
\hline Amputation/hindquarter & $23(18)$ \\
\hline \multicolumn{2}{|l|}{ Margin in local recurrence surgery } \\
\hline Wide & $21(17)$ \\
\hline Marginal & $34(27)$ \\
\hline Intralesional & $7(4)$ \\
\hline No surgery & $40(32)$ \\
\hline Not reported & $14(11)$ \\
\hline Second local recurrence & $32 / 86(37)$ \\
\hline Metastasis prior or at the time of local recurrence & $44 / 126(35)$ \\
\hline Grade 1 & $0 / 27(0)$ \\
\hline Grade 2 & $22 / 47(47)$ \\
\hline Grade 3 & $11 / 29(38)$ \\
\hline Dedifferentiated & $11 / 23(48)$ \\
\hline Patients with metastases during the study time & $69(55)$ \\
\hline Mean time from primary operation to local recurrence, mths & 28 (2 to 237$)$ \\
\hline Mean time from first to second local recurrence, mths & $33(0$ to 208$)$ \\
\hline Mean age at the time of local recurrence, yrs & $56(13$ to 96$)$ \\
\hline
\end{tabular}

intralesional curettage gives good local and systemic control, but this is reliant on accurately differentiating grade $1 \mathrm{CS}$ from grade 2 CS preoperatively, as intralesional curettage is not an acceptable treatment for grade $2 \mathrm{CS}$, due to the high rate of local recurrence (LR) and a decreased survival after LR. ${ }^{11}$

Misdiagnosing the grade may lead to an underestimation of the required margin and thereby increase the risk of LR. In true grade $1 \mathrm{CS}$, LR has a low impact on survival. We have previously shown that in grade 2 and 3 CS, LR significantly decreased the disease-specific survival (DSS) in competing risk analysis, where synchronous metastases (metastases developed before LR, at the time of LR, or within 90 days after LR) and death due to other causes were considered to be competing events in analyses of the role of LR in DSS. ${ }^{11}$ High-grade tumours are easier to diagnose and the best surgical strategy is clearly defined. ${ }^{11,12}$

Wide surgical excision minimizes the possibility of LR, but is often mutilating and sometimes, depending on the location of the tumour, impossible to achieve. A positive margin is a well-documented risk for LR in CS, but in higher grades the definition for an acceptable wide margin remains a matter for debate. We have previously shown that surgeons should aim for a $4 \mathrm{~mm}$ margin to reduce the risk of LR in high grade CS. ${ }^{11}$

The purpose of the current study was to investigate the effect of surgical treatment of locally recurrent chondrosarcoma on local recurrence-free survival (LRFS) and DSS.

\section{Patients and Methods}

This retrospective study included 126 patients, identified from a prospectively maintained database, who had been diagnosed with LR of a chondrosarcoma of the pelvis or a limb bone between 1990 and 2015 at the Royal Orthopaedic Hospital, Birmingham, United Kingdom. There were 44 female patients $(35 \%)$ and 82 male patients $(65 \%)$ with a mean age at the time of LR of 56 years (13 to 96). The 126 patients represented $24.3 \%$ of the total number of patients with a primary CS (519) who had been treated during this time. All patients were diagnosed and treated at the Royal Orthopaedic Hospital. Those who were primarily treated elsewhere and referred for the management of a recurrence were excluded. Details of the clinical data collected at the time of primary tumour and LR included the site (appendicular, extremity, or pelvis); primary and LR tumour size (in centimetres); type of operation at the time of primary or LR (limb-salvage or amputation); surgical margin achieved at resection of the primary tumour and the LR; grade of the primary tumour and the LR; gender; age; and oncological outcomes, including LRFS and DSS. A minimum two years' follow-up and complete histopathology records were available for all patients included in the study. Resection and LR specimens were examined by specialized bone sarcoma pathologists, for grade and involvement of margins. The highest grade seen on histology was the grade recorded, even when this higher grade consisted of only a small number of cells. The margin was quantified by a specialist bone sarcoma pathologist and classified according to the system described by Enneking et al. ${ }^{13}$ Histological biopsies or specimens were not re-reviewed but the original report was respected.

Statistical analysis. Descriptive statistics were used to describe demographic data. The Kaplan-Meier method was used to determine LRFS and DSS. Survival rates were calculated from the date of LR to the most recent follow-up, confirmation of a second LR, or death. A Kruskal-Wallis test was used for statistical analysis of means between the groups. Univariable analysis was performed by comparing groups with log-rank test with subsequent multivariable Cox proportional hazard analysis of significant variables to identify predictors of LRFS and DSS. A p-value $<0.05$ was considered significant. All statistical analysis was completed using SPSS Statistics 24.0 (IBM Corp., Armonk, New York).

\section{Results}

Clinical features. The mean age for patients with synchronous metastases at the time of LR was 61 years (21 to 88). For those without metastases at the time of LR, the mean age was 53 years $(13$ to 84$)(p=0.004)$. The mean time from primary surgery to LR was 28 months ( 2 to 237). The mean follow-up after LR was 45 months (0 to 256). Patient characteristics are summarized in 


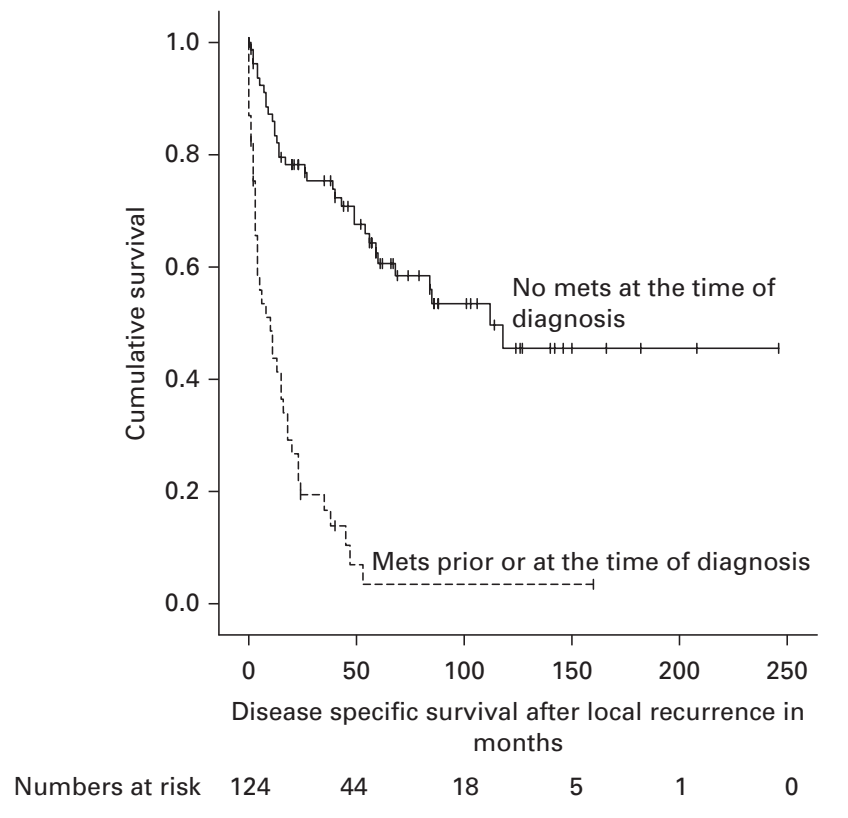

Fig. 1

Disease-specific survival after local recurrence stratified by metastases (mets) prior to or at the time of local recurrence.

Table I. The most common sites of the primary tumour were the proximal femur in 28 patients $(22.2 \%)$, the acetabulum in 24 patients $(19.0 \%)$, the ilium in 16 patients $(12.7 \%)$, the distal femur in 15 patients $(11.9 \%)$, the scapula in eight patients $(6.3 \%)$, the finger and proximal tibia in six patients $(4.8 \%)$, the hemipelvis and pubic bone in five patients $(4.0 \%)$, the proximal humerus in four patients $(3.2 \%)$, the sacrum and ischial bone in three patients $(2.4 \%)$, the proximal fibula in two patients $(1.6 \%)$, and the distal tibia in one patient $(0.8 \%)$.

Altogether, 44 of the 126 patients $(35 \%)$ had metastases prior to, or at the time of LR. Metastasis was a significant negative factor for survival. Survival for patients with metastases prior to, or at the time of, LR was $43.7 \%$ at one year and $6.9 \%$ at five years: $50.0 \%$ of patients died within eight months of developing metastases (Fig. 1). Surgical treatment of LR among patients with metastases at the time of or prior to LR does not improve survival ( $p=0.218$ ) (Fig. 2).

The histological grade of the LR was available for 81 patients $(64 \%)$. In 18 of these 81 patients $(22 \%)$, the grade of LR had increased from the original resection specimen. Of 18 grade 1 primary tumours, eight had increased to grade 2 in the LR specimen, three had increased to grade 3 , and one had increased to a dedifferentiated (DD) CS. Of 33 grade 2 primary CS, five had increased to grade 3 in the LR specimen and two had increased into DD CS. The grade of LR remained the same in all grade 3 and DD CS. In grade $1 \mathrm{CS}$ primary tumours treated by intralesional curettage, the increase in grade did not have an impact on overall survival $(\mathrm{p}=0.238)$.

Of the 126 patients, 40 patients (32\%) did not undergo surgery for their LR. In most, due to the presence of metastatic disease prior to, or at the time of, LR, 28 of the 126 patients $(22 \%)$ underwent amputation (including hindquarter amputation). Of

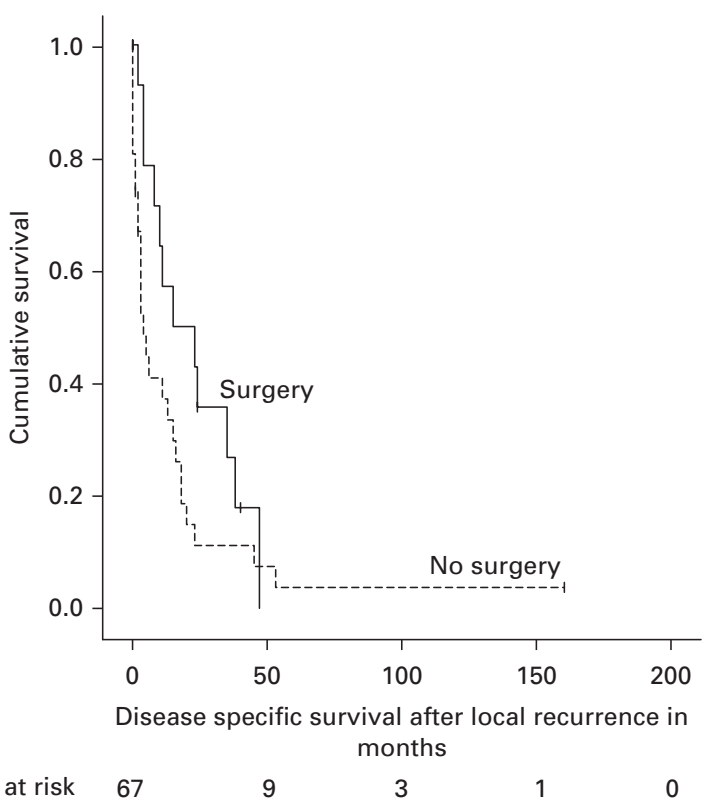

Fig. 2

Disease-specific survival after local recurrence stratified by surgery for local recurrence in patients with metastases.

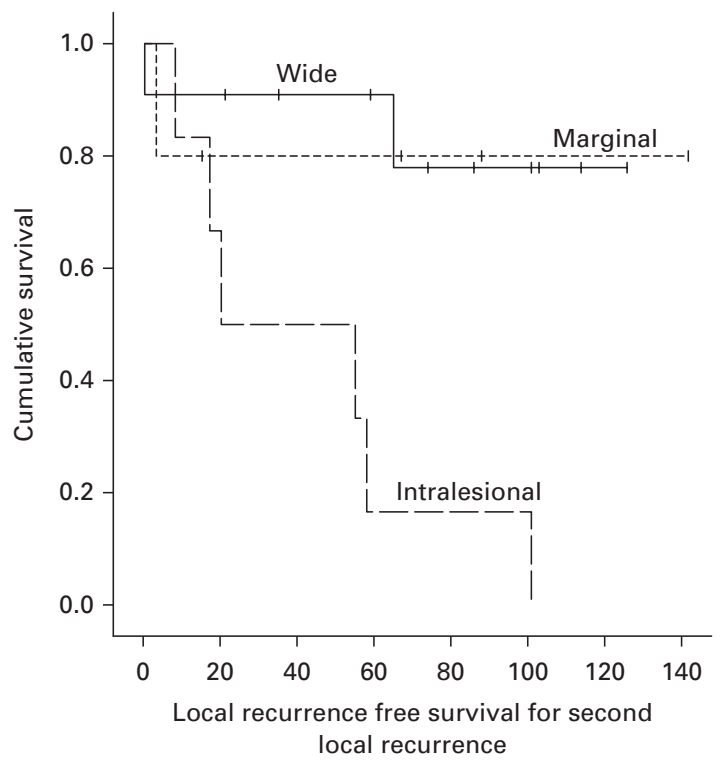

Numbers at risk

$\begin{array}{llllllll}22 & 18 & 17 & 15 & 12 & 8 & 2 & 1\end{array}$

Fig. 3

Local recurrence-free survival stratified by margin.

the 126 patients, 58 patients $(46 \%)$ had limb salvage or local resection of the LR at the site of a previous amputation. Margin $(p=0.251)$, DSS $(p=0.091)$, and LRFS $(p=0.117)$ were unaffected by the method of surgical management of the LR in terms of limb salvage or limb sacrifice. Surgical margin of the LR resection specimen was recorded in 72 patients $(83.7 \%)$ and was intralesional in 17 patients $(23.6 \%)$, marginal in 34 patients $(47.2 \%)$ and wide in 21 patients $(29.2 \%)$. A second LR 


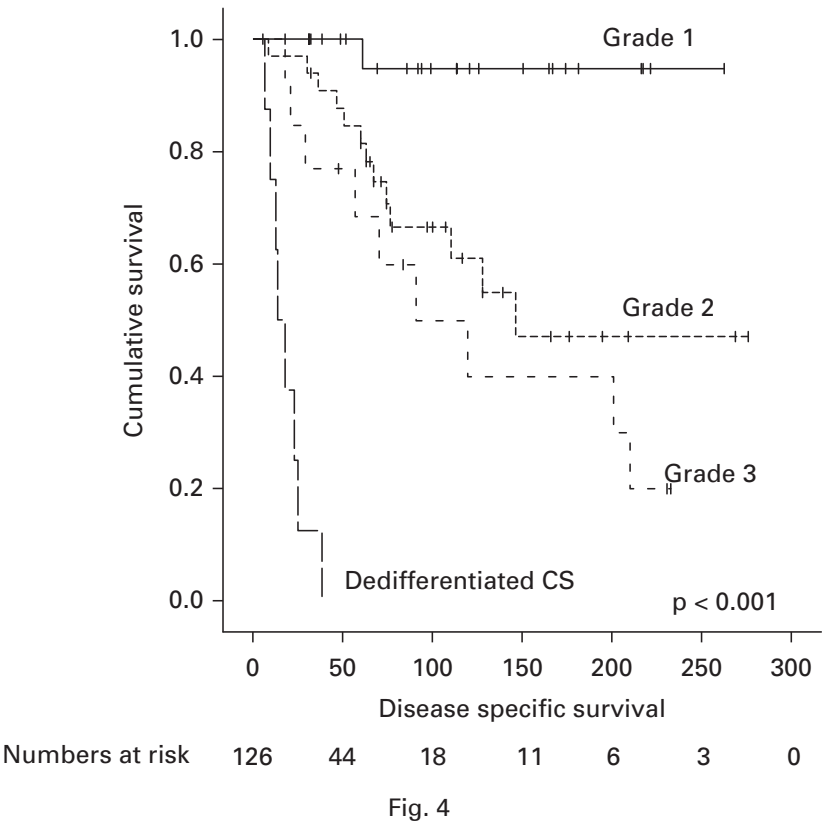

Disease-specific survival after local recurrence stratified by grade.

Table II. Results from univariable analysis for disease-specific survival in patients without metastases prior to, or at the time of, local recurrence

\begin{tabular}{lr}
\hline Variable & p-value \\
\hline Second local recurrence & 0.606 \\
Wide margin & 0.044 \\
Metastases & $<0.001$ \\
Grade & $<0.001$ \\
Gender & 0.136 \\
Location (pelvic vs extremity) & 0.456 \\
Increase of grade in local recurrence & 0.584 \\
\hline
\end{tabular}

*Kaplan-Meier analysis

was seen in 32 patients $(37.2 \%)$. The mean time from the first LR to subsequent LR was 33 months (1 to 208). LRFS for the second LR (LRFS) was $65.7 \%$ at five years and $42.6 \%$ at ten years. A wide margin was a good predictor in LRFS for second LR in univariable analysis when compared with intralesional margin $(p=0.002)$ but marginal margin did not reach statistical significance when compared with intralesional margin (p = 0.084) (Fig. 3).

Overall, DSS after LR was $42.1 \%$ at five years and $31.2 \%$ at ten years. For patients without metastases prior to, or at the time of, LR it was $62.5 \%$ and $45.5 \%$, respectively (Fig. $1)$. In patients without metastases prior to, or at the time of, LR, significant factors affecting DSS after univariable analysis were grade $(\mathrm{p}<0.001)$ (Fig. 4), wide margin compared with intralesional margin $(\mathrm{p}=0.044)$, wide margin compared with marginal margin $(\mathrm{p}=0.026)$, and marginal margin compared with intralesional margin $(\mathrm{p}=0.046)$ in resection of the LR (Fig. 5). After multivariable analysis, grade, age at diagnosis of LR, and time from index surgery to LR were significant factors for DSS. These results are summarized in Tables II and III.

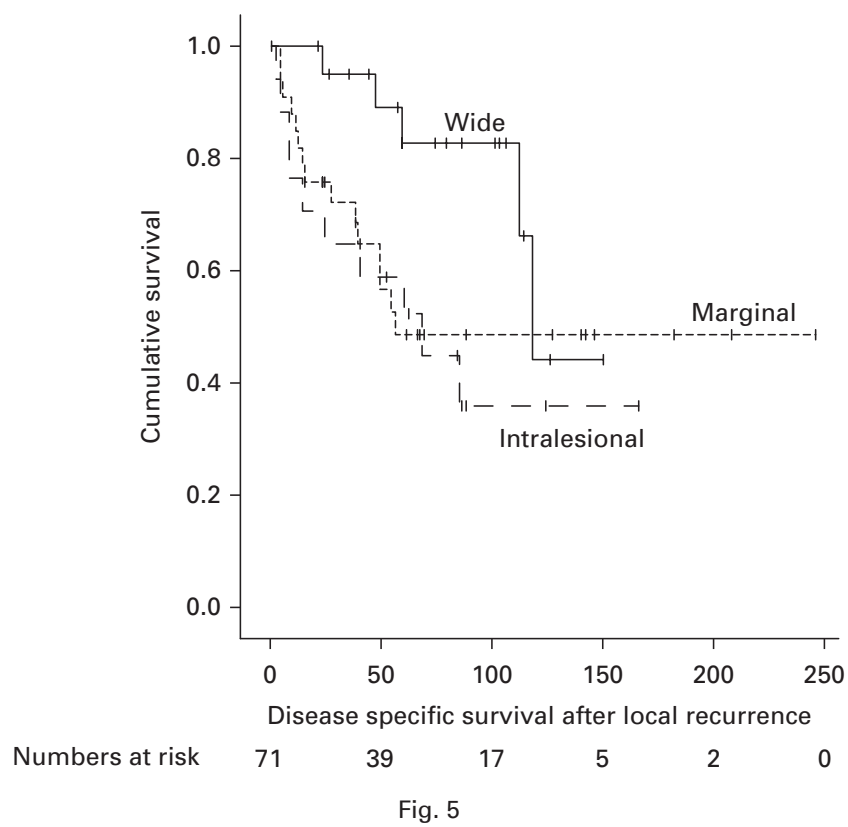

Disease-specific survival after local recurrence stratified by margin.

Table III. Predictors in multivariate analysis for disease-specific survival in patients without metastases prior to, or at the time of, local recurrence

\begin{tabular}{llll}
\hline & $\begin{array}{l}\text { Hazard } \\
\text { ratio }\end{array}$ & $\begin{array}{l}\mathbf{9 5 \%} \text { confidence } \\
\text { interval }\end{array}$ & p-value \\
\hline Dedifferentiated CS & 1 & & \\
Grade 1 CS & 0.003 & 0.000 to 0.050 & 0.000 \\
Grade 2 CS & 0.018 & 0.002 to 0.205 & 0.006 \\
Grade 3 CS & 0.23 & 0.002 to 0.299 & 0.004 \\
Age at the time of surgery, per year & 1.028 & 1.005 to 1.051 & 0.018 \\
Time to LR in months, per month & 0.942 & 0.891 to 0.995 & 0.034 \\
Progression in grade of LR & 0.757 & 0.276 to 2.072 & 0.587 \\
Location (extremity vs pelvis) & 0.710 & 0.368 to 1.370 & 0.307 \\
\hline
\end{tabular}

*Cox regression analysis

CS, chondrosarcoma; LR, local recurrence

\section{Discussion}

Recurrence of CS, whether in the form of local or metastatic disease, adversely affects survival. The aim of treatment of a primary CS is to avoid LR, but in cases of LR, we have shown that for patients without metastases at the time of LR, resection of recurrent disease with wide margins improves both DSS and LRFS, when compared with marginal or intralesional margins. Further surgery may lead to additional morbidity and a higher rate of amputation. Although amputation as a method of treatment of the primary tumour itself does not increase control of systemic or local disease, ${ }^{11}$ large resections and reconstructions in the primary operation may reduce the possibility of further limb salvage surgery, thereby increasing the risk of higher amputation or hind-/forequarter amputation.

The definition of wide margin in Enneking's classification system refers to removing the involved part of the bone with a cuff of normal tissue. The metric definition of an acceptable margin has never been accurately defined. The incidence of wide resection margins in our study was considerably lower 
than that reported in the literature, being only $29 \%$ when compared with $76 \%$ in the study from Streitbuerger et al. ${ }^{14}$ However, the survival rates were almost identical, which highlights the difficulty and the discrepancy that exists in defining what constitutes a wide margin or, more importantly, an adequate margin to reduce the risk of LR. ${ }^{14}$

The DSS after LR for all patients was $42.1 \%$ at five years and $31.2 \%$ at ten years. For patients without metastases prior to, or at the time of, LR, the DSS was $62.5 \%$ and $45.5 \%$ at five and ten years, respectively. Survival rates are slightly lower than in some studies in the literature, ${ }^{15,16}$ but in agreement with other studies. ${ }^{14,17}$ In line with current studies in the literature, tumour grade was the most important prognostic factor for patient survival in LRs. ${ }^{6,12,13,18,19}$ We found that LR of grade 1 CS was associated with a substantially worse DSS only if the grade of LR had progressed. The difference was not statistically significant, although this may be compounded by the rarity of the event. The role of progression of grade in LR has long been debated. ${ }^{17,20-22}$ We have previously shown that the grade of CS should be defined by the highest grade seen in the specimen regardless of how small the area of involvement. ${ }^{12}$ We would speculate that higher grade areas seen in the LR are reflective of the difficulty of grading the primary tumour, as small areas of higher grade may be overlooked when analyzing the primary specimen.

The development of metastasis is a poor prognostic indicator; $50 \%$ of patients with metastases prior to, or at the time of, LR died within eight months, with most of the rest succumbing to the disease within five years, irrespective of the grade. Despite the potential bias towards more aggressive treatment for patients without disseminated disease, surgery for LR in patients with metastases prior to, or at the time of, LR did not give any statistically significant survival advantage. We would therefore advocate that aggressive mutilating surgery should not be offered to patients in the presence of metastatic disease, unless this is within the context of a palliative approach. The role of metastasectomy for patients with resectable metastases and LR was not investigated in this study but may be considered on a case-by-case basis. However, the development of metastatic disease with LR is suggestive of a more aggressive form of the disease and so caution should be exercised as to the achievement of cure with combined resection.

Primary pelvic CS is notoriously difficult to treat; treatment has a worse outcome than that of extremity CS. ${ }^{1,18}$ In accordance with current literature, our results show that late age at onset and shorter disease-free interval are additional factors which adversely affect the outcome after LR. ${ }^{17}$ Therefore, the early and accurate detection of LR by frequent follow-up with local imaging is of paramount importance. Whether the frequency and modality of this follow-up should be tailored on an individual patient basis based on the grade, site, size, and margin remains an area of debate. In the absence of such data, regular surveillance in line with national and international guidelines should be advocated.

This study has a number of limitations. First, it is a retrospective study compiled from a prospectively maintained database from a single hospital. Second, margin determinations were recorded from pathology reports rather than re-analyzing each specimen. Therefore, the data relating to margin is prone to sampling error not only of the margin but also of the grade of the tumour at resection.

In conclusion, aggressive surgical resection with wide margins remains the cornerstone for achieving disease control in patients with locally recurrent $\mathrm{CS}$ without metastatic disease, to ensure long-term systemic and local DSS.

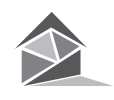

Take home message

- The development of local recurrence (LR) in chondrosarcoma (CS) has a detrimental effect on overall survival, although this is affected by the grade of the original tumour and the grade of the LR.

- Surgery for patients with LR of CS who have previously developed metastases or develop them at the time of the recurrence does not improve overall survival.

- Resection of recurrent disease improves overall survival and diseasespecific survival in patients without metastatic disease, only when resection achieves a wide margin.

\section{Twitter}

Follow M. K. Laitinen@MinnaLaitinen2

Follow the authors @ROHOncology

\section{References}

1. Björnsson J, McLeod RA, Unni KK, Ilstrup DM, Pritchard DJ. Primary chondrosarcoma of long bones and limb girdles. Cancer 1998;83:2105-2119.

2. Giuffrida AY, Burgueno JE, Koniaris LG, et al. Chondrosarcoma in the United States (1973 to 2003): an analysis of 2890 cases from the SEER database. J Bone Joint Surg [Am] 2009;91-A:1063-1072

3. Skeletal Lesions Interobserver Correlation among Expert Diagnosticians (SLICED) Study Group. Reliability of histopathologic and radiologic grading of cartilaginous neoplasms in long bones. J Bone Joint Surg [Am] 2007;89-A: 2113-2123.

4. Stihsen C, Panotopoulos J, Puchner SE, et al. The outcome of the surgical treatment of pelvic chondrosarcomas: a competing risk analysis of 58 tumours from a single centre. Bone Joint J 2017;99-B:686-696.

5. Fiorenza F, Abudu A, Grimer RJ, et al. Risk factors for survival and local control in chondrosarcoma of bone. J Bone Joint Surg [Br] 2002;84-B:93-99.

6. Angelini A, Guerra G, Mavrogenis AF, et al. Clinical outcome of central conventional chondrosarcoma. J Surg Oncol 2012;106:929-937.

7. Andreou D, Gilg MM, Gosheger G, et al. Metastatic potential of grade I chondrosarcoma of bone: results of a multi-institutional study. Ann Surg Oncol 2016;23:120-125

8. Campanacci DA, Scoccianti G, Franchi A, et al. Surgical treatment of central grade 1 chondrosarcoma of the appendicular skeleton. J Orthop Traumatol 2013;14:101-107

9. Verdegaal SH, Brouwers HF, van Zwet EW, Hogendoorn PC, Taminiau AH. Low-grade chondrosarcoma of long bones treated with intralesional curettage followed by application of phenol, ethanol, and bone-grafting. J Bone Joint Surg [Am] 2012;94-A:1201-1207.

10. Funovics PT, Panotopoulos J, Sabeti-Aschraf M, et al. Low-grade chondrosarcoma of bone: experiences from the Vienna Bone and Soft Tissue Tumour Registry. Int Orthop 2011;35:1049-1056.

11. Stevenson JD, Laitinen MK, Parry MC, et al. The role of surgical margins in chondrosarcoma. Eur J Surg Oncol 2018;44:1412-1418.

12. Laitinen MK, Stevenson JD, Parry MC, et al. The role of grade in local recurrence and the disease-specific survival in chondrosarcomas. Bone Joint $J$ 2018;100-B:662-666.

13. Enneking W, Dunham W, Gebhardt M, Malawar M, Pritchard D. A system for the classification of skeletal resections. Chir Organi Mov 1990;75(Suppl):217-240.

14. Streitbuerger A, Ahrens $\mathbf{H}$, Gosheger $\mathbf{G}$, et al. The treatment of locally recurrent chondrosarcoma: is extensive further surgery justified? J Bone Joint Surg $[\mathrm{Br}]$ 2012;94-B:122-127.

15. Lin PP, Alfawareh MD, Takeuchi A, Moon BS, Lewis V0. Sixty percent 10-year survival of patients with chondrosarcoma after local recurrence. Clin Orthop Relat Res 2012;470:670-676. 
16. Kim HS, Bindiganavile SS, Han I. Oncologic outcome after local recurrence of chondrosarcoma: Analysis of prognostic factors. J Surg Oncol 2015;111:957-961.

17. Schwab JH, Wenger D, Unni K, Sim FH. Does local recurrence impact survival in low-grade chondrosarcoma of the long bones? Clin Orthop Relat Res 2007;462:175-180

18. Andreou D, Ruppin S, Fehlberg S, et al. Survival and prognostic factors in chondrosarcoma: results in 115 patients with long-term follow-up. Acta Orthop 2011:82:749-755.

19. Gelderblom H, Hogendoorn PC, Dijkstra SD, et al. The clinical approach towards chondrosarcoma. Oncologist 2008;13:320-329.

20. Weber KL, Pring ME, Sim FH. Treatment and outcome of recurrent pelvic chondrosarcoma. Clin Orthop Relat Res 2002;397:19-28.

21. Donati D, El Ghoneimy A, Bertoni F, Di Bella C, Mercuri M. Surgical treatment and outcome of conventional pelvic chondrosarcoma. J Bone Joint Surg $[\mathrm{Br}]$ 2005;87-B:1527-1530.

22. Lee FY, Mankin HJ, Fondren G, et al. Chondrosarcoma of bone: an assessment of outcome. J Bone Joint Surg [Am] 1999;81-A:326-338.
Author contributions:

M. K. Laitinen: Study conception and design, Collected and analyzed the data, Prepared the manuscript.

M. C. Parry: Study conception and design, Prepared the manuscript.

L-R. Le Nail: Collected and analyzed the data, Prepared the manuscript.

C. H. Wigley: Collected and analyzed the data, Prepared the manuscript.

J. D. Stevenson: Prepared the manuscript.

L. M. Jeys: Study conception and design, Prepared the manuscript.

\section{Funding statement:}

No benefits in any form have been received or will be received from a commercial party related directly or indirectly to the subject of this article.

Acknowledgements:

The authors would like to thank the pathologists V. Sumathi, L-G. Kingblom, and D. C. Mangham.

This article was primary edited by A. C. Ross. 